\title{
Síndrome de Burnout em docentes universitários: mapeamento e reflexões acerca do
}

\section{trabalho}

\author{
Burnout syndrome in university professors: mapping and reflections on work \\ Síndrome de Burnout en profesores universitarios: mapeo y reflexiones sobre el trabajo
}

Alexa Maria Carneiro de Oliveira

ORCID: https://orcid.org/0000-0001-5329-1049 Universidade Federal da Paraíba, Brasil

Email: alexamcarneiro@gmail.com

Lidiane Firmino Dantas

ORCID: https://orcid.org/0000-0002-1917-3265 Universidade Federal da Paraíba, Brasi Email: 1diane78@gmail.com

Danilo Nascimento Rolim dos Santos

ORCID: https://orcid.org/0000-0002-3076-6360 Universidade Federal da Paraíba, Brasil

Email: danilonrs@hotmail.com

Angelica Barros Araújo

ORCID: https://orcid.org/0000-0001-7029-9667 Universidade Federal da Paraíba, Brasil Email: angelicabarros12@ hotmail.com

Mairana Maria Angélica Santos

ORCID: https://orcid.org/0000-0002-9782-9426 Universidade Federal da Paraíba, Brasil mairanam@outlook.com

Bárbara Iansã de Lima Barroso

ORCID: https://orcid.org/0000-0002-3591-4350 Universidade Federal da Paraíba, Brasil barbarabarroso@yahoo.com.br

\begin{abstract}
Resumo
Objetivo: identificar a presença da Síndrome de Burnout em docentes universitários no contexto das relações de saúde, trabalho e doença. Métodos: estudo transversal descritivo, no qual utilizou-se do questionário JBEILI, elaborado e adaptado por Chafic Jbeili, inspirado no Maslach Burnout Inventory, adaptada e validada, utilizando a estratégia do Websurveys a partir de um formulário implantado na plataforma Google Forms. Amostra constituída por 108 docentes, distribuídos entre 11 Departamentos. Foi aplicado o teste comparativo de Kruskal-Wallis, o teste de Levene, o teste Shapiro-Wilk e o teste de comparações múltiplas. Resultados: participaram do estudo 108 docentes de instituição pública de ensino superior, sendo 88,8\% mulheres e 11,2\% homens, distribuídos em 11 departamentos, dos quais, três destes apresentaram elevada taxa de sinais para a síndrome na fase inicial. Conclusão: identificou-se que os profissionais dos Departamentos de Enfermagem, Odontologia e Terapia Ocupacional estão na fase inicial da Síndrome de Burnout. Palavras-chave: Burnout; Saúde do trabalhador; Docentes; Trabalho precário; Estresse ocupacional.
\end{abstract}

\begin{abstract}
Objective: to identify the presence of Burnout Syndrome in university professors in the context of health, work and illness relationships. Methods: descriptive cross-sectional study, in which the JBEILI questionnaire was used, developed and adapted by Chafic Jbeili, inspired by the Maslach Burnout Inventory, adapted and validated, using the Websurveys strategy from a form implemented in the Google Forms platform. Sample constituted by 108 professors, distributed among 11 Departments. The Kruskal-Wallis comparative test, the Levene test, the Shapiro-Wilk test and the multiple comparisons test were applied. Results: 108 professors from a public higher education institution participated in the study, $88.8 \%$ women and $11.2 \%$ men, distributed in 11 departments, three of which had a high rate of signs for the syndrome in the initial phase. Conclusion: it was identified that professionals from the Departments of Nursing, Dentistry and Occupational Therapy are in the initial stage of Burnout Syndrome.
\end{abstract}

Keywords: Burnout; Worker's health; Teachers; Precarious work; Occupational stress.

\section{Resumen}

Objetivo: identificar la presencia del Síndrome de Burnout en profesores universitarios en el contexto de las relaciones salud, trabajo y enfermedad. Métodos: estudio descriptivo transversal, en el que se utilizó el cuestionario JBEILI, 
desarrollado y adaptado por Chafic Jbeili, inspirado en el Maslach Burnout Inventory, adaptado y validado, utilizando la estrategia Websurveys desde un formulario implementado en la plataforma Google Forms. Muestra constituida por 108 profesores, distribuidos entre 11 Departamentos. Se aplicó la prueba comparativa de Kruskal-Wallis, la prueba de Levene, la prueba de Shapiro-Wilk y la prueba de comparaciones múltiples. Resultados: participaron del estudio 108 profesores de una institución pública de educación superior, 88,8\% mujeres y $11,2 \%$ hombres, distribuidos en 11 departamentos, tres de los cuales tenían una alta tasa de signos del síndrome en la fase inicial. Conclusión: se identificó que los profesionales de los Departamentos de Enfermería, Odontología y Terapia Ocupacional se encuentran en la etapa inicial del Síndrome de Burnout.

Palabras clave: Burnout; Salud del trabajador; Maestros; Trabajo precario; Estrés laboral.

\section{Introdução}

Burnout é uma expressão originada na língua inglesa, sendo descrita como “queima após desgaste”. Retrata a perda da função devido à processo de exaustão. Tal expressão passou a representar o sofrimento dos trabalhadores em seu contexto laboral, relacionado à grande falta de motivação e diminuição da satisfação em exercer suas atividades de trabalho, decorrente da intensa sensação de exaustão. Desse modo, sua denotação significa “estar esgotado” ou “queimado” (Maslach \& Jackson, 1981).

A definição amplamente divulgada cientificamente sobre a Síndrome de Burnout respalda-se na compreensão social psicológica de Maslach e Jackson, a qual descreve a Síndrome de Burnout como uma resposta à tensão emocional recorrente ao lidar demasiadamente com indivíduos no ambiente de trabalho (Fernandes, 2020; Maslach, Schaufeli, \& Leiter MP, 2001).

Comumente, a Síndrome de Burnout caracteriza-se como um transtorno adaptativo às repetitivas situações de estresse, correlacionando-se as condições e exigências de trabalho. O seu avanço costuma ser insidioso e normalmente despercebido pelo trabalhador, além de apresentar manifestações múltiplas, sendo a mais frequente o cansaço emocional (Fernandes, 2020).

De acordo com o quantitativo de Docentes em processo de adoecimento no trabalho, a Síndrome de Burnout possui uma severidade no que tange o processo de adoecimento desses profissionais, em deterimento a outros trabalhadores, apresentando uma alteração do nível de saúde desta população (Maslach \& Jackson, 1981; Leite \& Nogueira, 2017).

Penteado e Souza Neto (2019) constroem seus argumentos dentro de uma perspectiva pautada no fazer Docente, onde a docência pode ser vista como o ato de descrever e avaliar atividades materiais e simbólicas, onde, o local de trabalho exerce uma função sobre ele próprio, suas expertises, suas experiências profissionais e de vida - sua identidade, associadas aos dispositivos da organização do trabalho e suas interferências, constroem o perfil do Docente. Tais autores afirmam que para compreender a atividade docente é preciso levar em consideração a integralidade dos elementos que compõem o trabalho, isto é: as redes, o lócus da educação, os arranjos, os indivíduos, os instrumentos, as finalidades, os métodos, os conhecimentos e as tecnologias, associadas a prática do ensino.

O termo docência tem sido tradicionalmente utilizado para representar o trabalho do professor. O fazer do Docente no ensino superior se efetiva pela propriedade com que este, enquanto mediador e transmissor de conhecimentos, reproduz seus saberes, no qual o seu maior papel é ensinar, difundir e incentivar o pensamento crítico e a inovação, porém existe um conjunto de funções que ultrapassam o exercício da docência, adentrando o sistema organizacional das Universidades (Galdino et al., 2021; Leite \& Nogueira, 2017).

No âmbito do sistema organizacional, acredita-se que o Docente no ensino superior realiza: atividade de ensino, de acordo com o projeto pedagógico do curso; domine os assuntos que envolve a sua área de atuação; domine e utilize diversas metodologias de processo de ensino; estruturar, preparar e ministrar aulas, além do quantitativo de horas para conseguir realizar atividades de monitoria, extensão e pesquisa, participando de ações e de atividades administrativas (Penteado \& Neto, 2019).

Em contrapartida a diversas funções exercidas pelo Docente, evidências científicas dos últimos dez anos, apresentam dados crescentes sobre o sofrimento dessa classe profissional em relação ao processo de proletarização que o iguala ao trabalhador do chão de fábrica, desmerecendo o processo intelectual dos Docentes e seus anos de formação. Dessa forma, o 
arranjo do trabalho desse profissional vem sofrendo um parâmetro caracterizado pela produção de influência capitalista, que resulta em competitividade excessiva, aumento da importância dos interesses individuais que vão além dos interesses dos trabalhadores, perpassando pelo sistema Governamental e Administrativo dos órgãos superiores, como Ministério da Educação (Galdino et al., 2021; Lambdin-Pattavina \& O’Brien, 2021; Peres, Marin, Tonhom, \& Marques, 2018; Cozby, 2003).

Para Leite e Nogueira (2017), o processo de sistematização do exercício Docente, na atualidade, tem particularidades que os submetem a situações estressantes no trabalho que, se constantes, levam à Síndrome de Burnout, a qual compromete o espaço de trabalho interferindo nos processos educacionais, podendo levar tais trabalhadores a um processo de estresse contínuo, de alienação, de desumanização e de apatia, elevando as chances de terem problemas relacionadas a saúde física, emocional, permanência no trabalho, além do absenteísmo (Galdino et al., 2021; Leite, \&Nogueira, 2017; Peres, Marin, Tonhom, \& Marques, 2018)

Sendo assim, são notórias as profundas alterações que os profissionais vivenciam no cenário atual do país, evidenciando a necessidade de novos estudos que possibilitem maior visibilidade às demandas relacionadas à saúde dos docentes da educação superior no Brasil (Jbeili, 2008; Souza et al., 2017). Face ao apresentado, o objetivo dessa pesquisa é identificar a presença da Síndrome de Burnout em docentes universitários no contexto das relações de saúde, trabalho e doença.

\section{Métodos}

Estudo transversal descritivo realizado em uma instituição pública de ensino superior localizada no Nordeste brasileiro, entre agosto de 2018 a junho de 2019.

Para a realização do cálculo amostral, foi considerado todos os Docentes de cursos de graduação ativos no Centro de Ciências da Saúde incluindo os departamentos de Enfermagem, Enfermagem Clínica, Enfermagem Saúde Coletiva, Farmácia, Fisiologia e Patologia, Fisioterapia, Fonoaudiologia, Morfologia, Nutrição, Odontologia Clinica e Social, Odontologia Restauradora e Terapia Ocupacional em uma totalidade de 349 docentes, sendo, uma estimativa de prevalência de 50\%, recomendado quando não se sabe previamente a prevalência real de se ter ou não a condição desejada a investigar, estimando-a então como aleatória.

Ainda, podendo haver uma taxa de perda de até $50 \%$ da amostra, no que tange ao perfil amostral por conveniência, descrito por Cozby (2003), o qual busca identificar dois perfis mais utilizados nas pesquisas - probabilidade e conveniência, seguindo esse parâmetro, foi considerado um nível de confiança de $95 \%$ e margem de erro de 5\%, resultando num tamanho mínimo da amostra de 108 participantes. Tal proporção foi estipulada em concordância com um conjunto de pesquisas prévias analisadas e identificadas na literatura nacional sobre o tema pesquisado.

Para o critério de inclusão foi considerado: ser docente em exercício, por no mínimo 6 meses que antecederam a coleta, estando em atividade de ensino, pesquisa e extensão. E o critério de exclusão: docentes com vínculo temporário como visitante e/ou substituto e colaboradores.

A coleta de dados foi realizada por meio de e-mail. Foi aplicado um instrumento com informações gerais (sexo, idade, tempo na instituição) e questões sobre as atividades desenvolvidas na instituição.

Para analisar a Síndrome de Burnout, foi aplicado o questionário JBEILI, elaborado e adaptado por Chafic Jbeili, inspirado no Maslach Burnout Inventory (MBI), utilizado de maneira informativa. Em estudo realizado por Carlotto e Câmara (2004) em uma capital do sul do Brasil, numa amostra que contemplou 563 docentes incluindo nível fundamental, médio e superior, o MBI em sua versão brasileira se apresentou de forma válida e confiável, possuindo as condições necessárias para ser utilizado no âmbito docente.

O questionário é ordenado em 20 alternativas variando de 1 a 5 , a qual indica a frequência em que os indivíduos experimentaram o conteúdo indicado pelo item, enumerados da seguinte forma: 01 - Nunca, 02- Anualmente, 03- Mensalmente, 
04- Semanalmente, 05- Diariamente, avaliando três componentes: exaustão emocional, despersonalização e a realização profissional. Nesse sentido, os scores é calculado através da soma dos resultados do questionário JBEILI, que possui a pontuação de 0 a 100, na qual de 0 a 20 se refere a nenhum indício de Burnout; de 21 a 40, possibilidade de desenvolver o Burnout - alerta para prevenções; de 41 a 60, fase inicial do Burnout, apresentando sintomas específicos; 61 a 80, o Burnout começa a se instalar e à ajuda profissional é necessária para combater agravamentos dos sintomas; e, entre 81 a 100, se instala a fase alarmante do Burnout, na qual necessita-se acompanhamento profissional com urgência (Beer \& Bianchi, 2017; Penteado \& Neto, 2019; Jbeili, 2008; Costa, 2018).

O Termo de Consentimento Livre e Esclarecido foi disponibilizado no e-mail enviado e o participante só teve acesso aos instrumentos caso concordasse em participar da pesquisa.

Os dados coletados foram digitados em planilha do programa Microsoft Office Excel ${ }^{\circledR}$ mediante dupla digitação e validação. Estes foram importados para o software The R Project for Statistical Computing 3.5.3, pacote estatístico "lavaan", e submetidos à análise estatística descritiva com medidas de frequência absoluta e relativa para caracterização dos participantes e dos dados referentes a escala de burnout, além de tendência central (média, mediana, máximo e mínimo) e de dispersão (desviopadrão) para idade.

O projeto foi submetido e aprovado pelo Comitê de Ética da Universidade Federal da Paraíba com o número do Certificado de Apresentação para Apreciação Ética (CAAE): 92650318.7.0000.5188 e Número do Parecer: 3.339.767. Todos os aspectos éticos foram contemplados para sua realização no Brasil segundo as Resoluções 466/2012 e 510/2016.

\section{Resultados}

Participaram do estudo 108 docentes do Centro de Ciencias da Saúde, sendo 88,8\% mulheres e 11,2\% homens, distribuídos em 11 departamentos.

Primeiramente, para a decisão de tratamento da amostra, verificou-se a homogeneidade das variâncias e a distribuição dos dados (teste de aderência). O teste de Levene indicou que as variâncias são homogêneas entre os grupos (Estatística=1,76; valor $\mathrm{p}=0,159)$. O teste de Shapiro-Wilk foi aplicado em cada grupo (tabela1) e no conjunto total de dados, rejeitando a hipótese de que todos os grupos departamentais apresentam o mesmo score (Estatística=8,524; valor p=0,036).

Tabela 1 - Resultado do teste de normalidade. João Pessoa-PB, Brasil, 2021.

\begin{tabular}{lcc}
\hline Grupo & Estatística & Valor $\boldsymbol{p}$ \\
\hline Enfermagem & 0,867 & 0,019 \\
Odontologia & 0,958 & 0,442 \\
Terapia Ocupacional & 0,902 & 0,033 \\
Demais Cursos & 0,969 & 0,233 \\
\hline
\end{tabular}

Fonte: Autores.

Admitindo-se a hipótese de que os dados são não paramétricos, o teste de Kruskall-Wallis (comparações múltiplas) foi executado entre os grupos departamentais estudados e como resultado apresentou diferença significativa apenas entre o grupo de Terapia Ocupacional e aquele de Enfermagem (Estatística=-29,89; valor p=0,047). As comparações entre os demais grupos não foram significativas (Kruskall-Wallis; p<0,05). 
Não foi observada diferença significativa entre os grupos de indivíduos com sexo diferentes quando se considera o escore resultante da soma, com base no teste de Mann-Whitney (Estatística=959,00; valor p=0,69).

Tabela 2 - Número de indivíduos em cada uma das fases de desenvolvimento da Síndrome de Burnout por departamento. João Pessoa-PB, Brasil, 2021.

\begin{tabular}{|c|c|c|c|c|c|}
\hline Departamento & Nenhum indício & Fase inicial & Instalação & Possibilidade & Considerável \\
\hline Enfermagem & 0 & 1 & 0 & 0 & 0 \\
\hline Enfermagem Clínica & 0 & 2 & 0 & 7 & 0 \\
\hline Enfermagem Saúde Coletiva & 0 & 2 & 2 & 3 & 0 \\
\hline Farmácia & 0 & 2 & 2 & 1 & 0 \\
\hline Fisiologia e Patologia & 0 & 1 & 1 & 3 & 0 \\
\hline Fisioterapia & 0 & 5 & 1 & 5 & 0 \\
\hline Fonoaudiologia & 0 & 11 & 1 & 1 & 0 \\
\hline Morfologia & 0 & 5 & 1 & 1 & 0 \\
\hline Nutrição & 0 & 2 & 0 & 4 & 0 \\
\hline Odontologia Clínica e Social & 0 & 7 & 3 & 6 & 0 \\
\hline Odontologia Restauradora & 0 & 1 & 0 & 5 & 0 \\
\hline Terapia Ocupacional & 0 & 18 & 4 & 0 & 0 \\
\hline
\end{tabular}

Fonte: Autores.

Devido ao número variável de participantes em muitos departamentos, agrupou-se os indivíduos daqueles em um único grupo. Assim, obtiveram-se quatro grupos departamentais, onde foi possível fazer uma comparação quanto ao nível de risco, apresentados a seguir (Tabela 3). Na Tabela 3, observa-se que os docentes do Departamento de Terapia Ocupacional, estão na fase inicial da Síndrome de Burnout, correspondendo $(81,82 \%)$ dos entrevistados desse departamento, o que representa um valor acima dos demais. Os maiores percentuais para os outros três grupos sob comparação ocorreram na fase da possibilidade de desenvolver a síndrome, que é uma fase mais preliminar. 
Tabela 3 - Percentual de indivíduos nas fases de adoecimento em quatro grupos de entrevistados. João Pessoa-PB, Brasil, 2021.

\begin{tabular}{|c|c|c|c|c|c|}
\hline Departamentos & Nenhum indício(\%) & Possibilidade(\%) & Fase inicial(\%) & Instalação(\%) & Considerável (\%) \\
\hline Enfermagem & 0,00 & 58,82 & 29,41 & 11,76 & 0,00 \\
\hline Odontologia & 0,00 & 50,00 & 36,36 & 13,64 & 0,00 \\
\hline Terapia Ocupacional & 0,00 & 0,00 & 81,82 & 18,18 & 0,00 \\
\hline Outros & 0,00 & 31,91 & 55,32 & 12,77 & 0,00 \\
\hline
\end{tabular}

Fonte: Autores.

\section{Discussão}

A maioria dos Docentes do departamento de Terapia Ocupacional, participantes deste estudo, apresentou pontuação elevada para a Síndrome de Burnout em fase inicial, de acordo com o instrumento utilizado. Conforme o JBEILI, nessa fase o Burnout começa a se instalar e a ajuda profissional é necessária para combater o agravamento dos sintomas.

Observa-se que o problema é alarmante quando se instigam indícios de desenvolvimento da Síndrome de Burnout no grupo estudado. Em todos os departamentos investigados não houve nenhum indivíduo sem qualquer indício de desenvolvimento da Síndrome (Tabela 2). Todos os participantes apresentaram sinais de que já se encontram em alguma das fases de desenvolvimento. Destes, $81,82 \%$ dos Docentes entrevistados, pertencem ao Departamento de Terapia Ocupacional e estão na fase inicial, já no Departamento de Odontologia o valor é de 36,36\% e na Enfermagem 29,41\%. Essa pontuação geral obtida conduz de forma significativa para a presença da fase inicial da Síndrome de Burnout nesse grupo de Docentes, confirmando evidências anteriores de trabalhadores da rede de saúde e assistência (Costa, 2018; Filha \& Morais, 2018).

O número elevado apresentado na fase inicial, repercute na vida profissional e na saúde do Docente, possuindo uma relação direta com a intensificação e volume da carga de trabalho, precarização do sistema organizacional, culminando no desencadeamento de estresse ocupacional, reduzindo a "capacidade do organismo" para o trabalho (Jbeili, 2008; Lusa, Martinelli, Moraes, \& Almeida, 2019; Costa, 2018; Filha \& Morais, 2018).

O trabalho concerne ao homem subsídios que permitem sua sobrevivência e supre suas principais necessidades, mas dentro de uma sociedade estruturada pelo capitalismo, existindo a necessidade de venda da sua força de trabalho, então convertida em mercadoria, modificando o sentido e a organização do trabalho (Cozby, 2003; Jbeili, 2008; Filha \& Morais, 2018).

Dejours (1991) retrata a pressão organizacional como um gerador constante de sofrimento psíquico, onde o sistema não admite que o trabalhador possua desejo, necessidade, medo, ansiedade e/ou cansaço, banindo qualquer sentimento ou sintoma de ordem mental, trazendo o corpo físico para o nível do aceitável. Na concepção Dejours (1991), onde Marx (2015) traz a organização do trabalho como um veículo que transporta o desejo do outro, gerando um mecanismo controlador, opressor e limitante que, por fim, o trabalhador se sente habitado e alienado por um desejo que não o pertence, nos remetendo ao sistema neoliberal que toma corpo, sentido e fazer de uma das principais ocupações humanas - o trabalho (Filha \& Morais, 2018).

No campo da educação, Costa (2018), retrata como um produto comercial para o sistema capitalista, onde, a educação pertence ao elemento da produtividade. Ainda, no capitalismo, a mais-valia absoluta é compreendida como a exploração máxima da força física do trabalhador, já na educação, a mais-valia relativa seria aquela, onde a exploração se concentra na esfera da inteligência (Cozby, 2003; Jbeili, 2008; Filha \& Morais, 2018). 
A consequência da junção entre o mecanismo neoliberal - capitalista e a mais-valia absoluta, no campo da formação e da educação, é a geração de um processo de subordinação do Docente ao Capital, onde, a exigência pela polivalência, a maximização de horas de trabalho e o acúmulo de cargos, são demandas comuns nas salas de aulas brasileiras (Leite \& Nogueira, 2017; Costa, 2018; Lima, 2019; Filha \& Morais, 2018).

Nesse sentido, os dados obtidos nessa pesquisa, mostram que os docentes da Terapia Ocupacional são os trabalhadores mais propensos à fase inicial e possível instalação da Síndrome. Nisto, pode-se considerar que a causa pesquisada está associada com a própria caracterista do trabalho docente (assistência e poucos profissionais concursados), já que o departamento da Universidade pesquisada, possui 25 docentes, tendo em vista a extensa carga de horário distribuídas em atividades realizadas dentro e fora do espaço de aprendizagem - sala de aula (Costa, 2018; Filha \& Morais, 2018).

Pesquisas internacionais como as de Escudero-Escudero e colaboradores (2020) identificaram que dos 758 profissionais que atuavam em diferentes serviços de saúde da Espanha, o perfil amostral analisado, apresentou alta prevalência para a Síndrome de Burnout os participantes com contratos permanentes e aqueles com contratos temporários ou provisórios; 525 $(69,3 \%)$ terapeutas ocupacionais trabalhavam em regime permanente. No que conminou nos elevados níveis da Síndrome de Burnout (Penteado \& Neto, 2019; Baptista, Soares, Raad, \& Santos, 2019; Leite \& Nogueira, 2017; Peres, Marin, Tonhom, \& Marques, 2018; Lima, 2019).

De acordo com Lambdin-Pattavina e O'Brien (2021), os Terapeutas Ocupacionais Docentes, tendem a ter mais possibilidades de risco ao surgimento e evolução da Síndrome de Burnout, devido aos elementos de sobrecarga presentes na prática docente e da amplitude de ações da prática profissional do Terapeuta Ocupacional (Peres, Marin, Tonhom, \& Marques, 2018; Cozby, 2003; Costa, 2018).

Outro fator que impulsiona o acúmulo de trabalho nos Docentes de Terapia Ocupacional, são as atividades de supervisão e acompanhamento de estágio, onde, muitas vezes o Docente faz papel também de preceptor e de Terapeuta Ocupacional dentro dos serviços (Lambdin-Pattavina \& O’Brien, 2021; Pinheiro et al., 2020).

No campo da Odontologia, pesquisa nacional com Cirurgiões Dentistas Docentes apresentam altos índices de precarização no trabalho, devido às peculiaridades da prática profissional associada a degradação do sistema de ensino e saúde brasileiro, levando à uma baixa satisfação profissional e produtividade, e elevando os índices de absenteísmo (Baptista, Soares, Raad, \& Santos, 2019; Leite \& Nogueira, 2017; Pinheiro et al., 2020; Filha \& Morais, 2018).

Ainda, no campo Odontológico, é evidenciado na literatura científica nacional e internacional a existência de uma sobrecarga física, sendo um profissional vulnerável aos riscos ocupacionais que surgem das características da sua atividade produtiva. Nesse sentindo, os estressores ocupacionais possuem organização, natureza e intensidade diversa e precisam ser analisados e estudados de forma profunda (Pinheiro et al., 2020).

Um estudo nacional realizado com Cirurgiões Dentistas Docentes, apresentou índice elevado a propensão a Síndrome de Burnout. Segundo os autores, tal fato pode estar associado a sobrecarga de trabalho, múltiplas tarefas que devem ser desempenhadas pelos Cirurgiões Dentista Docentes, altas horas de trabalho, elevado fluxo de alunos e pacientes, adicionado às condições laborais das salas de aula, clínicas e laboratórios, que se constituiu o espaço de trabalho desse profissional (Pinheiro et al., 2020).

Os trabalhadores da área de Enfermagem, fazem parte de uma das classes trabalhadoras que mais adoencem mentalmente, sendo estes, suscetíveis ao aparecimento e desencadeamento da Síndrome de Burnout, pela característica laboral da sua atividade de trabalho, caracterizada pelo contato diário com indivíduos doentes; trabalho em turnos; relações interpessoais intensas e hierárquicas nos estabelecimentos de saúde, contribuindo para uma sobrecarga física, cognitiva e emocional desses trabalhadores (Baptista, Soares, Raad, \& Santos, 2019; Leite \& Nogueira, 2017). 
A aparição da Síndrome pode estar conectada a fatores predisponentes de origem da natureza do trabalho docente, das questões sociais que aflingem um país em desenvovlimento, como o Brasil e a questões de ordem pessoal, como, cobrança excessiva e competitividade. Tais questões predispostas precisam ser debatidas profundamente no âmbito da Síndrome de Burnout e os profissionais Docentes (Leite \& Nogueira, 2017; Lambdin-Pattavina \& O’Brien, 2021; Cozby, 2003; Jbeili, 2008; Costa, 2018).

Outra peculiaridade proeminente no cerne dessa pesquisa, é o fato de que os Docentes mais propensos ao surgimento e ao desencadeamento da Síndrome são profisisonais da área da saúde, estes, que possuem contato direto com a área assistencial (Galdino et al., 2021; Peres, Marin, Tonhom, \& Marques, 2018; Pinheiro et al., 2020; Escudero-Escudero, Segura-Fragoso, \& Cantero-Garlito, 2020).

Face ao exposto, é importante salientar que o aumento da precarização do trabalho nas Universidades possui uma associação lógica mercadológica, que corresponde com a ampliação dos contratos de trabalho de forma temporária, redução dos números de Docentes com dedicação exclusiva e com os elevados número de docentes substitutos. (Costa, 2018; Lima, 2019).

Para Souza e colaborades (2018) o trabalho docente passa por um processo de desvalorização, onde, evidencia-se o pouco reconhecimento social, baixos salários, gestão autoritária vinda da gestão superior, jornada extensa, formação deficitária e acúmulo de tarefas extraclasses. Ademais, a presença de infraestrutura precária, carência de recursos materiais e humanos cujo resultado é a sobrecarga de trabalho e maiores desdobramentos por parte do corpo docente (Costa, 2018; Lima, 2019).

Ainda, Souza e colaboradores (2018) em sua pesquisa, analisa o trabalho docente como uma atividade imaterial, na qual a atividade intelectual preenche a maior parte do tempo de produçãoo do Docente, com consequências físicas e mentais para estes trabalahdores. Corroborando com os estudos de Costa (2018) o qual relata que presença de indicadores de Burnout implica em prejuízos ao docente quanto ao planejamento de aula, alterações na motivação e na criatividade, baixas expectativas, distanciamento e diminuição da simpatia pelos alunos (Costa, 2018; Lima, 2019).

No que tange o ensino público no Brasil, as Universidades Federais têm como eixos principais a tríade ensino-pesquisaextensão, tornando possível uma relação mais estreita entre o mundo acadêmico e a sociedade, acrescentando a ambos aprendizados científicos e populares, sendo assim, o docente deve dispor tempo, planejamento e ações que visem os três pilares da educação superior, além de desempenhar cargos administrativos e de gestão (Leite \& Nogueira, 2017; Lusa, Martinelli, Moraes, \& Almeida, 2019; Costa, 2018; Filha \& Morais, 2018).

A Síndrome de Burnout é muitas vezes negligenciada por falta de diagnóstico padronizado e pelo acumulo de estresse crônico advindo do ambiente de trabalho, atigindo os processos relacionais, o rendimento e o funcionamento da organização como um todo (Maslach \& Jackson, 1981; Fernandes, 2020; Baptista, Soares, Raad, \& Santos, 2019; Leite \& Nogueira, 2017).

Os sinais e sintomas de burnout entre docentes deve ser considerado na atualidade como um problema social, que exige pesquisas mais aprofundadas e com amostras substanciais, uma vez que as consequências para o ensino e formação de jovens profissionais no país pode ser afetada devido acúmulo de trabalho, desvalorização, precarização e estresse ocupacional que inundam as universidades brasileiras (Lusa, Martinelli, Moraes, \& Almeida, 2019).

\section{Conclusão}

Identificou-se que os profissionais dos Departamentos de Enfermagem, Odontologia e Terapia Ocupacional estão na fase inicial da Síndrome de Burnout, indicando a possível necessidade de acompanhamento profissional para estes docentes. Esta pesquisa mostra-se relevante e contributiva para que o docente universitário possa fazer reflexões acerca do seu trabalho e sua qualidade de vida no contexto da docência, principalmente para a área de Saúde e Trabalho. Tendo em vista as múltiplas atividades de trabalho que são exigidas aos docentes, perante as recentes transformações no âmbito político-universitário no que 
concerne o aumento do produtivismo, competições interpessoais e demais agentes agressores que possam levar ao aparecimento da Síndrome de Burnout.

Por fim, este estudo pode ter limitações no que tange a amostra por departamentos. Os autores sugerem pesquisas mais amplas que abranja uma extensão da amostra, considerando as variáveis como regime de contrato, carga horária total de horas trabalhadas, prováveis diferenças entre instituições públicas e privadas, dados sociodemográficos, período e tempo de formação, bem como o detalhamento das experiências anteriores, para que os resultados possam ser generalizados.

\section{Referências}

Baptista M. N., Soares T. F. P., Raad A. J., \& Santos L. M. Burnout, estresse, depressão e suporte laboral em professores universitários. Rev Psicol Organ Trab. 2019; 19(1):564-570. http://dx.doi.org/10.17652/rpot/2019.1.15417

Beer L. T., \& Bianchi R. Confirmatory factor analysis of the Maslach Burnout Inventory. European Journal of Psychological https://econtent.hogrefe.com/doi/pdf/10.1027/1015-5759/a000392

Carlotto, M. S., \& Câmara, S. G. Análise fatorial do Maslach Burnout Inventory (MBI) em uma amostra de professores de instituições particulares. Psicologia em Estudo, 9(3), 499-505.

Cozby P. C. Métodos de pesquisa em ciências do comportamento. Atlas; 2003.

Costa A. As transformações no mundo do trabalho em tempos neoliberais e suas implicações no trabalho docente. Movimento-Revista de Educação. 2018; 5(8):281-312. https://doi.org/10.22409/mov.v5i8.431

Dejours C. A loucura do trabalho: estudo de psicopatologia do trabalho. S.1; 1991

Escudero-Escudero A. C., Segura-Fragoso A., \& Cantero-Garlito P.A. Burnout Syndrome in Occupational Therapists in Spain: prevalence and risk factors. International Journal of Environmental Research and Public Health. 2020; 17(9):1-8. https://doi.org/10.3390/ijerph17093164

Fernandes C. C. Fenómeno de burnout em fisioterapeutas: revisão bibliográfica. https://bdigital.ufp.pt/handle/10284/9111

Filha C. N. M. B. L., \& Morais A.N. Prevalência e fatores de risco do burnout nos docentes universitários. Revista Contemporânea de Educação. 2018; 13(27): 453-471. http://dx.doi.org/10.20500/rce.v13i26.13431

Galdino M. J. Q., Martins J. T., Robazzi M. L. C. C., Pelloso S. M., Barreto M. F. C., \& Haddad M. C. F. L. Burnout, workaholism e qualidade de vida entre docentes de pós-graduação em enfermagem. Acta Paulista Enfermagem. 2021; 34(eAPE00451):1-8. https://doi.org/10.37689/acta-ape/2021AO00451

Jbeili C. Questionário preliminar de identificação da burnout. http://www.chafic.com.br/index_arquivos/burnout.pdf

Lambdin-Pattavina C., \& O’Brien J. Teaching in the New Millennium: an autoethnographic approach to exploring occupational adaptation of occupational therapy professors. Journal of Higher Education Theory and Practice. 2021; 21(4):94-105. https://doi.org/10.33423/jhetp.v21i4.4211

Leite A. F., \& Nogueira J. A. D. Fatores condicionantes de saúde relacionados ao trabalho de professores universitários da área da saúde: uma revisão integrativa. Revista Brasileira de Saúde Ocupacional. 2017; 42(e6):1-15. http://dx.doi.org/10.1590/2317-6369000010116

Lima K. R. S. Educação superior em tempos de ajustes neoliberais e regressão de direitos. Revista Katálysis. 2019; 22(3):513-524. https://doi.org/10.1590/1982$02592019 v 22 n 3 p 525$

Lusa M. G., Martinelli T., Moraes S. A., \& Almeida T. P. A Universidade pública em tempos de ajustes neoliberais e desmonte de direitos. Revista Katálysis. 2019; 22(3):536-547. https://doi.org/10.1590/1982-02592019v22n3p536

Maslach C., \& Jackson S. E. The measurement of experienced burnout. Journal of Organizational Behavior. 1981; 2:99-113. http://dx.doi.org/10.1002/job.4030020205

Maslach C., Schaufeli W. B., \& Leiter M. P. Job Burnout. Annual Review of Psychology. 2001; 52(1):397-422. http://dx.doi.org/10.1146/annurev.psych.52.1.397

Penteado R.Z., \& Neto S. S. Mal-estar, sofrimento e adoecimento do professor: de narrativas do trabalho e da cultura docente à docência como profissão. Saúde e Sociedade. 2019; 28(1):135-153. https://doi.org/10.1590/S0104-12902019180304

Peres C. R. F. B., Marin M. J. S., Tonhom S. F. R., \& Marques M. L. S. F. Desafios atuais na formação do enfermeiro: olhar dos docentes. Rev Rene. 2018;19:e3160. https://doi.org/10.15253/2175-6783.2018193160

Pinheiro W. L. L, Júnior A. F. C., Junior C. S. B., da Conceição K. C. F., dos Santos I. O., \& Casseb T. F, et al. Estresse e síndrome de burnout em profissionais de odontologia. Revista Eletrônica Acervo Saúde. 2020; 51(e3270):1-10. http://dx.doi.org/10.25248/reas.e3270.2020

Souza K. R., Mendonça A. L. O., Rodrigues M. A. S., Felix E. G., Teixeira L. R., \& Santos M. B. M., et al. A nova organização do trabalho na universidade pública: consequências coletivas da precarização na saúde dos docentes. Ciênc Saúde Coletiva. 2017; 22(11):3667-3676. 10.1590/1413-812320172211.01192016 